\title{
Experimental effects of the off-shell structure in anomalous neutral triple gauge vertices
}

\author{
J. Alcaraz \\ CERN, 1211 Genève 23, Switzerland \\ and CIEMAT, Avenida Complutense 22, 28040 Madrid, Spain
}

(Received 22 November 2001; published 1 April 2002)

\begin{abstract}
We discuss differences between on-shell and off-shell treatments in the search for anomalous neutral triple gauge couplings in $e^{+} e^{-}$collisions. We find that the usual on-shell framework represents an optimal starting point, covering all scenarios in which a reasonable experimental sensitivity is expected. We show that off-shell effects lead to negligible deviations at the experimental level, provided that $e^{+} e^{-} \rightarrow f \bar{f} \gamma$ and $e^{+} e^{-} \rightarrow f \bar{f} f^{\prime} \bar{f}^{\prime}$ analyses are performed in regions where $Z^{*} \rightarrow f \bar{f}, f^{\prime} \bar{f}^{\prime}$ production is dominant. For consistency reasons, we advocate the use of a natural extension of the on-shell definitions, which takes into account the correct off-shell dependences. Contrary to what has been recently suggested in the literature, we find that no $S U(2)_{L} \times U(1)_{Y}$ constraints among neutral triple gauge couplings can be imposed in a general case.
\end{abstract}

DOI: 10.1103/PhysRevD.65.075020

\section{INTRODUCTION}

The measurement of triple gauge boson couplings is one of the main items in the physics program of present and future colliders [1]. In this context, anomalous neutral triple gauge couplings (NTGC), which are not present in the standard model (SM) at the tree level, constitute an interesting possibility for new physics [2]. Fermilab Tevatron [3,4] and CERN $e^{+} e^{-}$collider (LEP) experiments [5-8] have carried out systematic searches for $Z V V$ couplings, where $V$ denotes any of the two SM neutral gauge bosons ( $Z$ or $\gamma$ ).

Recently it has been claimed $[9,10]$ that off-shell effects in anomalous couplings cannot be ignored, and that the spectrum of possible coupling structures may be larger. LEP analyses on the search for anomalous off-shell couplings have followed [6]. The aim of this paper is to clarify the situation concerning the different NTGC sets and conventions, and the implications of these choices on present experimental limits.

The study is organized as follows. The first section introduces the usual convention employed in the search for anomalous NTGCs. Next we present a general discussion on NTGCs arising at the lowest order in $\sqrt{s} / \Lambda$, where $\Lambda$ represents the scale of new physics. A new convention for the NTGC structures will be suggested at this stage. It will be shown that the new convention should lead to no changes in present experimental results [3-8]. A different approach will be used in order to build up the off-shell dependences for the remaining (higher order) NTGCs. The study will be completed with a short discussion on the experimental consequences of imposing $S U(2)_{L} \times U(1)_{Y}$ SM symmetry constraints. The conclusions are presented in the last section.

\section{THE STANDARD CONVENTION: ON-SHELL ANOMALOUS COUPLINGS}

The usual definition of anomalous NTGCs is obtained from the vertex structures (see Fig. 1):
PACS number(s): 12.60.Cn, 13.10.+q, 13.38.Dg

$$
\begin{aligned}
\Gamma_{\mathrm{Z} \gamma V}^{\alpha \beta \mu}= & i e \frac{q_{V}^{2}-m_{V}^{2}}{m_{\mathrm{Z}}^{2}}\left\{h_{1}^{V}\left(q_{\gamma}^{\mu} g^{\alpha \beta}-q_{\gamma}^{\alpha} g^{\beta \mu}\right)\right. \\
& +h_{2}^{V} \frac{q_{V}^{\alpha}}{m_{\mathrm{Z}}^{2}}\left(q_{\gamma} q_{V} g^{\beta \mu}-q_{\gamma}^{\mu} q_{V}^{\beta}\right)+h_{3}^{V} \epsilon^{\alpha \beta \mu \rho} q_{\gamma \rho} \\
& \left.+h_{4}^{V} \frac{q_{V}^{\alpha}}{m_{\mathrm{Z}}^{2}} \epsilon^{\mu \beta \rho \sigma} q_{V \rho} q_{\gamma \sigma}\right\}
\end{aligned}
$$

for the $e^{+} e^{-} \rightarrow Z \gamma$ case, and

$$
\begin{aligned}
\Gamma_{\mathrm{Z}_{1} \mathrm{Z}_{2} V}^{\alpha \beta \mu}= & i e \frac{q_{V}^{2}-m_{V}^{2}}{m_{\mathrm{Z}}^{2}}\left\{f_{4}^{V}\left(q_{V}^{\alpha} g^{\beta \mu}+q_{V}^{\beta} g^{\mu \alpha}\right)\right. \\
& \left.+f_{5}^{V} \epsilon^{\alpha \beta \mu \rho}\left(q_{\mathrm{Z}_{1} \rho}-q_{\mathrm{Z}_{2} \rho}\right)\right\}
\end{aligned}
$$

for the $e^{+} e^{-} \rightarrow Z Z$ case. The momenta of the particles in the vertex are denoted by $q_{V}$ (ingoing) and $q_{\mathrm{Z}}, q_{\gamma}, q_{\mathrm{Z}_{1}}, q_{\mathrm{Z}_{2}}$ (outgoing). The electromagnetic coupling, $e=\sqrt{4 \pi \alpha}$, and the $Z$ mass, $m_{\mathrm{Z}}$, appear as arbitrary constant factors.

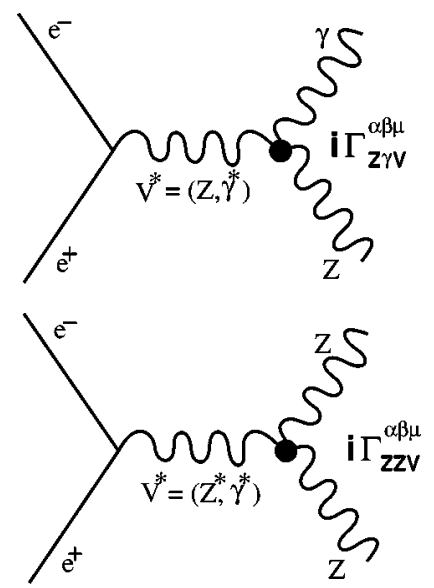

FIG. 1. Anomalous vertex structures for $Z \gamma V$ (left) and $Z Z V$ (right) anomalous couplings. 
The anomalous $Z \gamma V$ couplings $h_{1}^{V}, h_{2}^{V}(V=Z, \gamma)$ correspond to $C P$ violating terms, whereas $h_{3}^{V}, h_{4}^{V}$ are related to $C P$ conserving ones. The anomalous $Z Z V$ couplings $f_{4}^{V}$ lead to $C P$ violating interactions, whereas $f_{5}^{V}$ are associated to a $C P$ conserving structure. All terms violate charge conjugation.

Both parametrizations were proposed for the first time in [11]. For the $e^{+} e^{-} \rightarrow Z \gamma$ case, the original proposal had to be modified [12] (an extra $i$ factor was included) in order to work with Hermitian Lagrangians for real values of the anomalous couplings.

The previous vertex expressions are the most general ones preserving Lorentz and electromagnetic gauge invariance, assuming that the bosons in the final state are on shell. Let us comment on some features related to the arbitrary factors in the convention:

The strength of the coupling is assumed to be electromagnetic, but it should be substituted in general by a coupling $g$, of order one:

$$
e \rightarrow g \sqrt{4 \pi}
$$

$h_{1}^{V}, h_{3}^{V}, f_{4}^{V}, f_{5}^{V}$ are accompanied by a $m_{\mathrm{Z}}^{-2}$ factor. They correspond to vertices arising from Lagrangians of dimension six or higher. It is convenient to reinterpret them in terms of the new physics scale $\Lambda$ :

$$
\frac{e}{m_{\mathrm{Z}}^{2}} \rightarrow \frac{g \sqrt{4 \pi}}{\Lambda^{2}}
$$

$h_{2}^{V}, h_{4}^{V}$ are accompanied by a $m_{\mathrm{Z}}^{-4}$ factor, and only appear via Lagrangian terms of dimension eight or higher. Similar to the previous case, the $m_{\mathrm{Z}}^{-4}$ dimensional factor could be substituted by $\Lambda^{-4}$ :

$$
\frac{e}{m_{\mathrm{Z}}^{4}} \rightarrow \frac{g \sqrt{4 \pi}}{\Lambda^{4}}
$$

Since this is just a matter of convention, adopted by all experiments until now, we are not proposing a redefinition in terms of scales of new physics. We just point out that if the sensitivities to $h_{1}^{V}, h_{3}^{V}$ and $h_{2}^{V}, h_{4}^{V}$ at center-of-mass energies $\sqrt{s} \gtrsim m_{\mathrm{Z}}$ turn out to be quite similar this is an artifact of the $m_{Z}^{2}$ factors in the convention. The actual sensitivity to the new physics scale $\Lambda$ is reduced in general for the higher dimension terms associated with $h_{2}^{V}, h_{4}^{V}$.

In general, all these couplings behave as complex form factors, with a dependence on $\sqrt{s}$. That is the case of the SM and of the minimal supersymmetric standard model (MSSM) close to the electroweak scale [2,10]. In the case of new physics at a scale $\Lambda \gg \sqrt{s}$ the imaginary parts and $\sqrt{s}$ dependences can be ignored, since they are suppressed by powers of $\left(s / \Lambda^{2}\right)^{n}$.

\section{OFF-SHELL COUPLINGS}

At the lowest dimension (six), only the following operators contain sensible ${ }^{1} Z V V$ vertex information:

$$
\begin{aligned}
& \mathcal{O}_{6}^{A}=\widetilde{Z}_{\mu \nu}\left(\partial_{\rho} Z^{\rho \mu}\right) Z^{\nu} \\
& \mathcal{O}_{6}^{B}=\widetilde{F}_{\mu \nu}\left(\partial_{\rho} Z^{\rho \mu}\right) Z^{\nu} \\
& \mathcal{O}_{6}^{C}=\widetilde{Z}_{\mu \nu}\left(\partial_{\rho} F^{\rho \mu}\right) Z^{\nu} \\
& \mathcal{O}_{6}^{D}=\widetilde{F}_{\mu \nu}\left(\partial_{\rho} F^{\rho \mu}\right) Z^{\nu} \\
& \widetilde{\mathcal{O}}_{6}^{A}=Z_{\mu \nu}\left(\partial_{\rho} Z^{\rho \mu}\right) Z^{\nu} \\
& \widetilde{\mathcal{O}}_{6}^{B}=F_{\mu \nu}\left(\partial_{\rho} Z^{\rho \mu}\right) Z^{\nu} \\
& \widetilde{\mathcal{O}}_{6}^{C}=Z_{\mu \nu}\left(\partial_{\rho} F^{\rho \mu}\right) Z^{\nu} \\
& \widetilde{\mathcal{O}}_{6}^{D}=F_{\mu \nu}\left(\partial_{\rho} F^{\rho \mu}\right) Z^{\nu}
\end{aligned}
$$

where $F^{\mu \nu}$ and $Z^{\mu \nu}$ are the tensor fields associated with the photon and the $Z$ particle, respectively, and $\widetilde{F}^{\mu \nu}$ $\equiv \epsilon^{\mu \nu \rho \delta} F_{\rho \delta}$. These terms give rise to anomalous vertices which we will parametrize as follows:

$$
\begin{aligned}
& \Gamma_{\mathrm{ZZZ}}^{\alpha \beta \mu} \rightarrow i e \frac{f_{5}^{\mathrm{Z}}}{m_{\mathrm{Z}}^{2}}\left[q_{1}^{2} \epsilon^{\alpha \beta \mu \rho}\left(q_{2 \rho}-q_{3 \rho}\right)+q_{2}^{2} \epsilon^{\alpha \beta \mu \rho}\left(q_{3 \rho}-q_{1 \rho}\right)\right. \\
& \left.+q_{3}^{2} \epsilon^{\alpha \beta \mu \rho}\left(q_{1 \rho}-q_{2 \rho}\right)\right] \\
& \Gamma_{\mathrm{Z} \gamma \mathrm{Z}}^{\alpha \beta \mu} \rightarrow i e \frac{h_{3}^{Z}}{m_{\mathrm{Z}}^{2}}\left[\left(q_{3}^{2}-q_{1}^{2}\right) \epsilon^{\alpha \beta \mu \rho} q_{2 \rho}\right] \\
& \Gamma_{\mathrm{ZZ} \gamma}^{\alpha \beta \mu} \rightarrow i e \frac{f_{5}^{\gamma}}{m_{\mathrm{Z}}^{2}}\left[q_{3}^{2} \epsilon^{\alpha \beta \mu \rho}\left(q_{1 \rho}-q_{2 \rho}\right)\right] \\
& \Gamma_{\mathrm{Z} \gamma \gamma}^{\alpha \beta \mu} \rightarrow i e \frac{h_{3}^{\gamma}}{m_{\mathrm{Z}}^{2}}\left[q_{3}^{2} \epsilon^{\alpha \beta \mu \rho} q_{2 \rho}-q_{2}^{2} \epsilon^{\alpha \beta \mu \rho} q_{3 \rho}\right] \\
& \Gamma_{\mathrm{ZZZ}}^{\alpha \beta \mu} \rightarrow i e \frac{f_{4}^{Z}}{m_{\mathrm{Z}}^{2}}\left[-q_{1}^{2}\left(q_{1}^{\beta} g^{\mu \alpha}+q_{1}^{\mu} g^{\alpha \beta}\right)\right. \\
& -i e \frac{q_{2}^{2}\left(q_{2}^{\alpha} g\right.}{m_{\mathrm{Z}}^{2}}\left[\left(q_{3}^{2}-q_{1}^{2}\right)\left(g^{\alpha \beta} q_{2}^{\mu}-q_{2}^{\mu} g^{\alpha \beta}\right)-q_{3}^{2}\left(q_{3}^{\alpha} g_{2}^{\alpha \mu}\right)\right] \\
& \left.\left.\Gamma_{3}^{\alpha \beta}+q_{3}^{\beta} g^{\mu \alpha}\right)\right]
\end{aligned}
$$

\footnotetext{
${ }^{1} \partial_{\mu} Z^{\mu}$ terms are ignored. They are only relevant for off-shell decays into very massive fermions, like $Z^{*} \rightarrow t \bar{t}[11]$.
} 


$$
\begin{aligned}
& \Gamma_{\mathrm{ZZ} \gamma}^{\alpha \beta \mu} \rightarrow i e \frac{f_{4}^{\gamma}}{m_{\mathrm{Z}}^{2}}\left[-q_{3}^{2}\left(g^{\beta \mu} q_{3}^{\alpha}+g^{\mu \alpha} q_{3}^{\beta}\right)\right] \\
& \Gamma_{\mathrm{Z} \gamma \gamma}^{\alpha \beta \mu} \rightarrow i e \frac{h_{1}^{\gamma}}{m_{\mathrm{Z}}^{2}}\left[q_{2}^{2}\left(q_{3}^{\beta} g^{\mu \alpha}-q_{3}^{\alpha} g^{\beta \mu}\right)+q_{3}^{2}\left(q_{2}^{\mu} g^{\alpha \beta}-q_{2}^{\alpha} g^{\beta \mu}\right)\right]
\end{aligned}
$$

where the introduction of the $h_{1}^{V}, h_{3}^{V}, f_{4}^{V}, f_{5}^{V}$ parameters will be justified later. The (always outgoing) four-momenta $q_{j}$ $(j=1,3)$ refer to the particles appearing in the position $j$ of the $V_{1} V_{2} V_{3}$ label. The following index correspondence is assumed: $1 \leftrightarrow \alpha, 2 \leftrightarrow \beta, 3 \leftrightarrow \mu$. Terms proportional to $q_{1}^{\alpha}, q_{2}^{\beta}$ and $q_{3}^{\mu}$ are neglected.

When particles 1 and 2 are assumed to be on-shell bosons, the previous expressions become

$$
\begin{aligned}
& \Gamma_{\mathrm{ZZZ}}^{\alpha \beta \mu} \rightarrow i e \frac{f_{5}^{\mathrm{Z}}}{m_{\mathrm{Z}}^{2}}\left[\left(q_{V}^{2}-m_{\mathrm{Z}}^{2}\right) \epsilon^{\alpha \beta \mu \rho}\left(q_{1 \rho}-q_{2 \rho}\right)\right] \\
& \Gamma_{\mathrm{Z} \gamma \mathrm{Z}}^{\alpha \beta \mu} \rightarrow i e \frac{h_{3}^{Z}}{m_{\mathrm{Z}}^{2}}\left[\left(q_{V}^{2}-m_{\mathrm{Z}}^{2}\right) \epsilon^{\alpha \beta \mu \rho} q_{2 \rho}\right] \\
& \Gamma_{\mathrm{ZZ} \gamma}^{\alpha \beta \mu} \rightarrow i e \frac{f_{5}^{\gamma}}{m_{\mathrm{Z}}^{2}}\left[q_{V}^{2} \epsilon^{\alpha \beta \mu \rho}\left(q_{1 \rho}-q_{2 \rho}\right)\right] \\
& \Gamma_{\mathrm{Z} \gamma \gamma}^{\alpha \beta \mu} \rightarrow i e \frac{h_{3}^{\gamma}}{m_{\mathrm{Z}}^{2}}\left[q_{V}^{2} \epsilon^{\alpha \beta \mu \rho} q_{2 \rho}\right] \\
& \Gamma_{\mathrm{Z} \gamma \gamma}^{\alpha \beta \mu} \rightarrow i e \frac{h_{1}^{\gamma}}{m_{\mathrm{Z}}^{2}}\left[q_{V}^{2}\left(g^{\alpha \beta} q_{2}^{\mu}-g^{\beta \mu} q_{2}^{\alpha}\right)\right] \\
& \Gamma_{\mathrm{ZZ} \gamma}^{\alpha \beta \mu} \rightarrow i e \frac{f_{4}^{\gamma}}{m_{\mathrm{Z}}^{2}}\left[q_{V}^{2}\left(g^{\beta \mu} q_{V}^{\alpha}+g^{\mu \alpha} q_{V}^{\beta}\right)\right] \\
& \Gamma_{\mathrm{Z} \gamma \mathrm{Z}}^{\alpha \beta \mu} \rightarrow i e \frac{f_{\mathrm{Z}}^{\mathrm{Z}}}{m_{\mathrm{Z}}^{2}}\left[\left(q_{V}^{2}-m_{\mathrm{Z}}^{2}\right)\left(g^{\beta \mu} q_{V}^{\alpha}+g^{\mu \alpha} q_{V}^{\beta}\right)\right] \\
& m_{\mathrm{Z}}^{Z}\left[\left(q_{V}^{2}-m_{\mathrm{Z}}^{2}\right)\left(g^{\alpha \beta} q_{2}^{\mu}-g^{\beta \mu} q_{2}^{\alpha}\right)\right]
\end{aligned}
$$

where $q_{V} \equiv-q_{3}$ (ingoing four-momentum).

No new terms are found when the final on-shell particles are assumed to be 1 and 3, or 2 and 3 . $^{2}$ Structures (19)-(26) exhaust all the on-shell possibilities among neutral gauge

\footnotetext{
${ }^{2}$ The surviving terms differ by trivial interchanges of identical bosons indices.
}

bosons. Now the reason for introducing $h_{1}^{V}, h_{3}^{V}, f_{4}^{V}$ and $f_{5}^{V}$ becomes evident: all terms lead to the usual convention of Eqs. (1),(2) in the on-shell limit. This feature was also noticed in $[9,10]$.

As commented before, $h_{2}^{V}$ and $h_{4}^{V}$ couplings do not appear here because they are associated with Lagrangians of higher dimension. Concerning the most general off-shell vertex structures (11)-(18), some important comments are necessary:

(a) The introduction of the $h_{j}^{V}$ and $f_{j}^{V}$ couplings in this context implies a redefinition of the convention in present $e^{+} e^{-} \rightarrow Z Z$ and $e^{+} e^{-} \rightarrow Z \gamma$ analyses. However, the next sections will show that off-shell and on-shell expressions lead to similar results at the experimental level.

(b) The inclusion of off-shell structures is theoretically well motivated, but it does not imply that experiments should search for anomalous effects in regions with dominant offshell boson production. The maximal sensitivity is always provided by the analysis of $e^{+} e^{-} \rightarrow Z^{*} \gamma \rightarrow f \bar{f} \gamma$ and $e^{+} e^{-}$ $\rightarrow Z^{*} Z^{*} \rightarrow f \bar{f} f^{\prime} \bar{f}^{\prime}$ events in the vicinity of the $Z$ resonances, corresponding to a sensible signal definition of $Z \gamma$ and $Z Z$ final states. There, in addition, "signal" statistics is high and non-sensitive backgrounds are reduced.

(c) The standard $e^{+} e^{-} \rightarrow Z \gamma$ and $e^{+} e^{-} \rightarrow Z Z$ analyses cover all reasonable types of vertex structures. No additional samples are required in order to complete a search for anomalous effects at the lowest dimension (six). And these terms are guaranteed to be the ones which provide the largest effects from new physics lying above the center-of-mass energy of the collision: $\Lambda>\sqrt{s}$.

\section{ON-SHELL VERSUS OFF-SHELL AT THE EXPERIMENTAL LEVEL}

Comparing Eqs. (11)-(18) and Eqs. (19)-(26), the following conclusions are obtained:

The on-shell and off-shell vertex functions associated with $f_{5}^{\gamma}$ and $f_{4}^{\gamma}$ are identical.

The on-shell and off-shell vertex functions associated with $h_{1}^{\gamma}$ and $h_{3}^{\gamma}$ coincide in the case of real photon production $\left(q_{2}^{2}=0\right)$, i.e., in the relevant case of $e^{+} e^{-} \rightarrow Z^{*} \gamma$ production.

The on-shell and off-shell vertex functions associated with $h_{j}^{Z}, f_{j}^{Z}$ differ by additive terms of order $\left(q_{Z}^{2}-m_{Z}^{2}\right) /\left(q_{V}^{2}\right.$ $\left.-m_{Z}^{2}\right) \approx m_{Z} \Gamma_{Z} /\left(s-m_{Z}^{2}\right)$.

Therefore, the only relevant differences between the two sets of expressions appear for $f_{j}^{Z}$ and $h_{j}^{Z}$. These differences are expected to be qualitatively small, but a quantitative statement is absolutely necessary in order to assess the validity of present experimental searches.

In order to quantify the effects of an off-shell treatment on present LEP results [5-8], $100000 e^{+} e^{-} \rightarrow(Z / \gamma)^{*} \gamma \rightarrow f \bar{f} \gamma$ and $e^{+} e^{-} \rightarrow(Z / \gamma)^{*}(Z / \gamma)^{*} \rightarrow f \bar{f} f^{\prime} \bar{f}^{\prime}$ events at a center-ofmass energy of $\sqrt{s}=200 \mathrm{GeV}$ are generated. The values $h_{j}^{Z}, f_{j}^{Z}=0.25,0.5,1.0,2.0$ are considered. A more realistic experimental scenario is obtained by selecting events in which the two-fermion invariant masses, $m_{f \bar{f}}$, are consistent with 
TABLE I. Relative difference in the number of expected events, $\Delta N / N$, between off-shell and on-shell analyses at $\sqrt{s}=200 \mathrm{GeV}$. Different values of the $h_{j}^{Z}$ anomalous couplings are considered. Cuts on the fermion-pair invariant mass, $\left|m_{f \bar{f}}-m_{\mathrm{Z}}\right|<10 \mathrm{GeV}$, and on the photon polar angle, $\left|\cos \theta_{\gamma}\right|<0.9$, are applied.

\begin{tabular}{ll}
\hline \hline Coupling value & \multicolumn{1}{c}{$\frac{\Delta N}{N}$} \\
\hline$h_{1}^{Z}=0.25$ & $(0.9 \pm 0.2) \times 10^{-5}$ \\
$h_{1}^{Z}=0.5$ & $(3.1 \pm 0.5) \times 10^{-5}$ \\
$h_{1}^{Z}=1.0$ & $(0.8 \pm 0.1) \times 10^{-4}$ \\
$h_{1}^{Z}=2.0$ & $(1.4 \pm 0.2) \times 10^{-4}$ \\
$h_{3}^{Z}=0.25$ & $(0.3 \pm 0.2) \times 10^{-5}$ \\
$h_{3}^{Z}=0.5$ & $(2.1 \pm 0.5) \times 10^{-5}$ \\
$h_{3}^{Z}=1.0$ & $(0.7 \pm 0.1) \times 10^{-4}$ \\
$h_{3}^{Z}=2.0$ & $(1.3 \pm 0.2) \times 10^{-4}$ \\
\hline \hline
\end{tabular}

the $Z$ mass, $\left|m_{f \bar{f}}-m_{\mathrm{Z}}\right|<10 \mathrm{GeV}$. In addition, a cut on the polar angle of photons, $\left|\cos \theta_{\gamma}\right|<0.9$, is applied.

For the $h_{j}^{Z}$ case, the study is performed by a reweighting procedure according to the $e^{+} e^{-} \rightarrow(Z / \gamma)^{*} \gamma \rightarrow f \bar{f} \gamma$ anomalous matrix element, either under off-shell [Eqs. (11)-(18)] or under on-shell [11] assumptions.

A first observable sensitive to anomalous couplings is the total cross section. The relative differences between off-shell and on-shell cases are reported in Table I. These extremely small numbers are somehow expected, since off-shell deviations have similar sizes but different signs above and below the $Z$ mass.

Even if the differences in the total rate are negligible, experiments frequently combine cross section measurements and shape information in the full phase space. A powerful way to study the effect of the differences in shape is by analyzing the mean values of the optimal observables of the process. In the general case the differential cross section in the presence of an anomalous coupling $h$ can be expressed as follows:

$$
\left.\frac{d^{2} \sigma}{d O_{1} d O_{2}}\right|_{h}=\left.\frac{d^{2} \sigma}{d O_{1} d O_{2}}\right|_{h=0}\left(1+h O_{1}+h^{2} O_{2}\right)
$$

where the variables $O_{1}$ and $O_{2}$, also known as optimal $o b$ servables, are functions of the phase space variables of the event, with no explicit dependence on $h$. The previous equation guarantees that the maximal information on $h$ is obtained by a study of the event density as a function of the variables $O_{1}$ and $O_{2}$.

For small $C P$-conserving couplings, $h_{3}^{Z} \rightarrow 0$, only the $O_{1}$ variable contributes. In fact, in the limit of vanishing couplings the maximal sensitivity is obtained by a simultaneous measurement of the total cross section and of the mean value of $O_{1}$. For $C P$-violating couplings like $h_{1}^{Z}, O_{1}$ is not the relevant variable, since $C P$-violating and $C P$-conserving
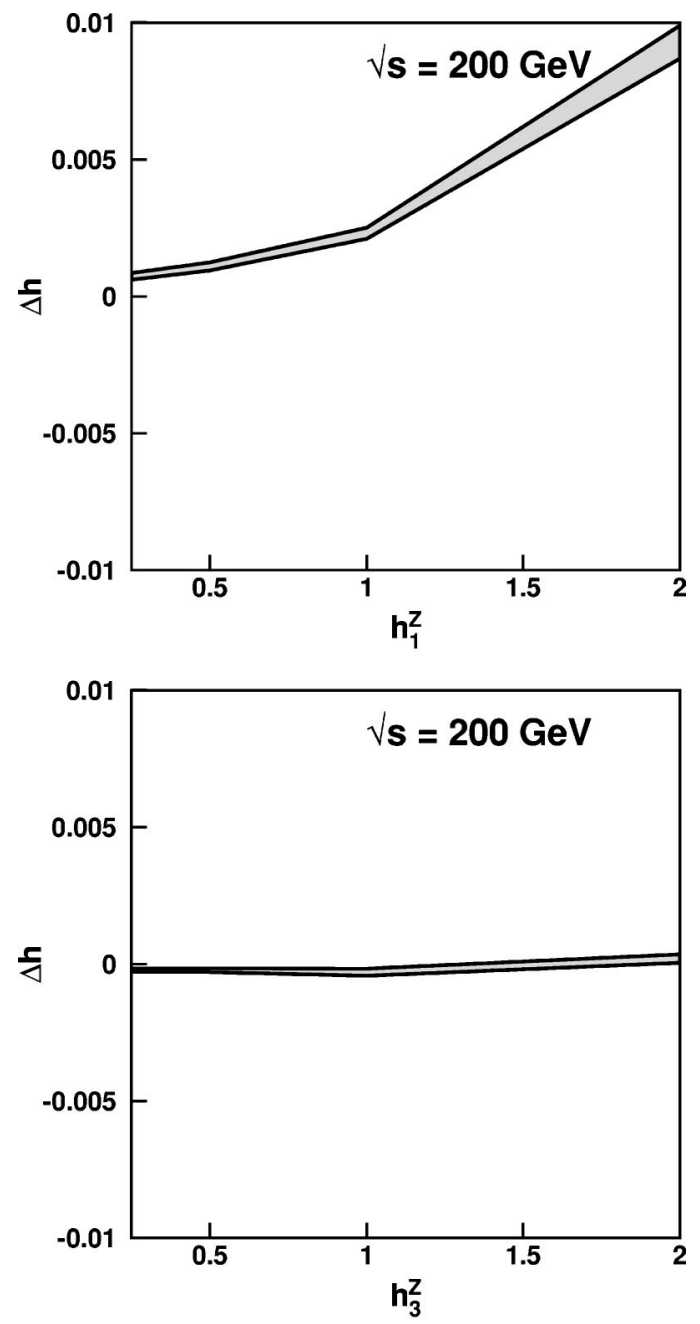

FIG. 2. Differences, $\Delta h$, between off-shell and on-shell measurements of the anomalous gauge couplings $h_{1}^{Z}$ (left) and $h_{3}^{Z}$ (right). Measurements are derived from the mean values of the $\mathrm{O}_{2}$ distribution (for $h_{1}^{Z}$ ) and of the $O_{1}$ distribution (for $h_{3}^{Z}$ ). The analyzed process is $e^{+} e^{-} \rightarrow(Z / \gamma)^{*} \gamma \rightarrow f \bar{f} \gamma$ at $\sqrt{s}=200 \mathrm{GeV}$.

(SM) terms do not interfere. ${ }^{3}$ In this case, $\mathrm{O}_{2}$ will be considered as the sensitive quantity.

Using the mean values of $O_{1}$ and $O_{2}$ as inputs, the values for the different couplings are extracted. The difference observed between the measurements of a coupling $h$ using offshell and on-shell approaches will be denoted by $\Delta h$. It quantifies the influence of discrepancies in the shapes of phase space distributions between the two treatments. As observed in Fig. 2, the absolute differences at $\sqrt{s}=200 \mathrm{GeV}$ never exceed $|\Delta h|=0.01$ in the range under study, and are negligible when compared to the present experimental uncertainties [13].

For the $f_{j}^{Z}$ case, the study is performed by a reweighting

\footnotetext{
${ }^{3}$ This is strictly true at the same order of perturbative expansion. In practice, some interference remains due to the presence of $i m_{\mathrm{Z}} \Gamma_{\mathrm{Z}}$ terms in the amplitudes, originating from higher order terms.
} 
TABLE II. Relative difference in the number of expected events, $\Delta N / N$, between off-shell and on-shell analyses at $\sqrt{s}$ $=200 \mathrm{GeV}$. Different values of the $f_{j}^{Z}$ anomalous couplings are considered. A cut on the relevant fermion-pair invariant masses, $\left|m_{f \bar{f}}-m_{\mathrm{Z}}\right|<10 \mathrm{GeV}$, is applied.

\begin{tabular}{lc}
\hline \hline Coupling value & $\frac{\Delta N}{N}$ \\
\hline$f_{4}^{Z}=0.25$ & $(0.6 \pm 0.1) \times 10^{-5}$ \\
$f_{4}^{Z}=0.5$ & $(2.1 \pm 0.2) \times 10^{-5}$ \\
$f_{4}^{Z}=1.0$ & $(5.8 \pm 0.5) \times 10^{-5}$ \\
$f_{4}^{Z}=2.0$ & $(1.1 \pm 0.1) \times 10^{-4}$ \\
$f_{5}^{Z}=0.25$ & $(5.4 \pm 0.9) \times 10^{-5}$ \\
$f_{5}^{Z}=0.5$ & $(1.7 \pm 0.2) \times 10^{-4}$ \\
$f_{5}^{Z}=1.0$ & $(5.3 \pm 0.3) \times 10^{-4}$ \\
$f_{5}^{Z}=2.0$ & $(1.5 \pm 0.1) \times 10^{-3}$ \\
\hline \hline
\end{tabular}
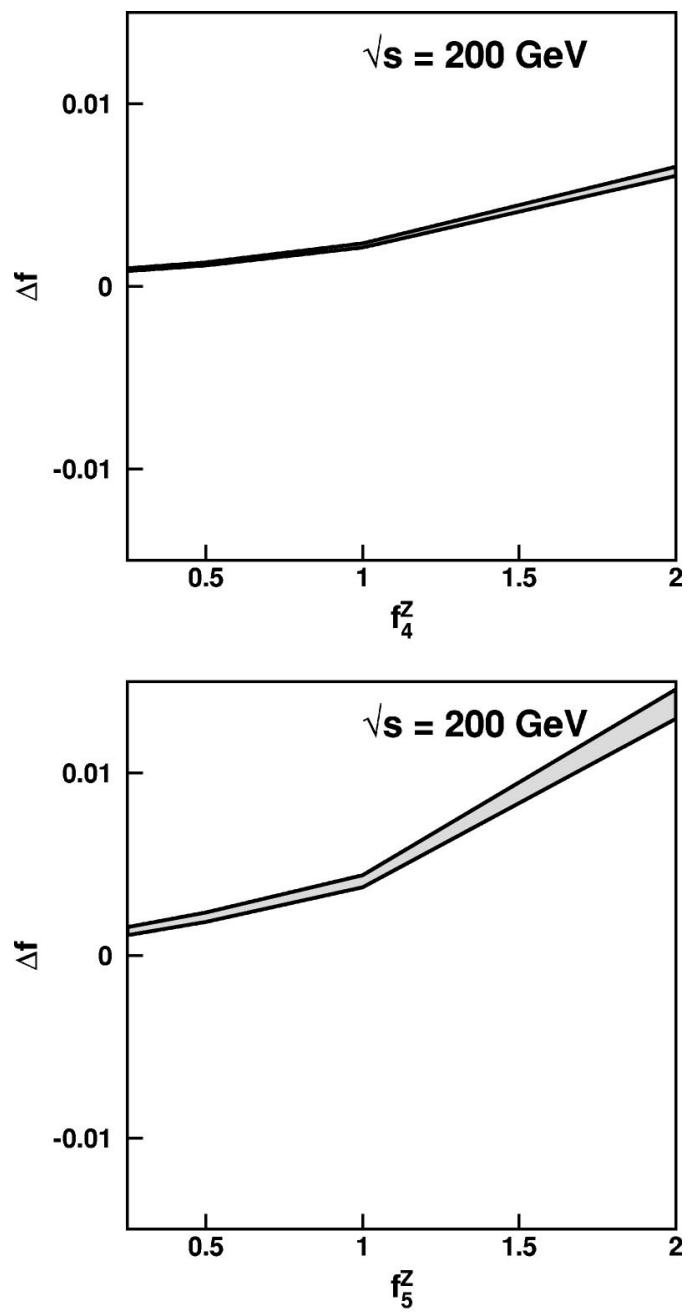

FIG. 3. Differences, $\Delta f$, between off-shell and on-shell measurements of the anomalous gauge couplings $f_{4}^{Z}$ (left) and $f_{5}^{Z}$ (right). Measurements are derived from the mean values of the $\mathrm{O}_{2}$ distribution (for $f_{4}^{Z}$ ) and of the $O_{1}$ distribution (for $f_{5}^{Z}$ ). The analyzed process is $e^{+} e^{-} \rightarrow(Z / \gamma)^{*}(Z / \gamma)^{*} \rightarrow f \bar{f} f^{\prime} \bar{f}^{\prime}$ at $\sqrt{s}=200 \mathrm{GeV}$.
TABLE III. Relative difference in the number of expected events, $\Delta N / N$, between off-shell and on-shell analyses at $\sqrt{s}=500$ $\mathrm{GeV}$. Different values of the $h_{j}^{Z}$ and $f_{j}^{Z}$ anomalous couplings are considered. Cuts on the fermion-pair invariant mass, $\left|m_{f \bar{f}}-m_{\mathrm{Z}}\right|$ $<10 \mathrm{GeV}$, and on the photon polar angle, $\left|\cos \theta_{\gamma}\right|<0.9$, are applied.

\begin{tabular}{lc}
\hline \hline & $\frac{\Delta N}{N}$ \\
Coupling value & $(0.4 \pm 0.1) \times 10^{-5}$ \\
\hline$h_{1}^{Z}=0.025$ & $(0.9 \pm 0.2) \times 10^{-5}$ \\
$h_{1}^{Z}=0.05$ & $(1.5 \pm 0.3) \times 10^{-5}$ \\
$h_{1}^{Z}=0.1$ & $(1.8 \pm 0.3) \times 10^{-5}$ \\
$h_{1}^{Z}=0.2$ & $(0.3 \pm 0.1) \times 10^{-5}$ \\
$h_{3}^{Z}=0.025$ & $(0.9 \pm 0.2) \times 10^{-5}$ \\
$h_{3}^{Z}=0.05$ & $(1.4 \pm 0.3) \times 10^{-5}$ \\
$h_{3}^{Z}=0.1$ & $-(0.8 \pm 0.9) \times 10^{-6}$ \\
$h_{3}^{Z}=0.2$ & $-(1.6 \pm 1.6) \times 10^{-6}$ \\
$f_{4}^{Z}=0.025$ & $-(2.1 \pm 2.0) \times 10^{-6}$ \\
$f_{4}^{Z}=0.05$ & $-(2.3 \pm 2.2) \times 10^{-6}$ \\
$f_{4}^{Z}=0.1$ & $(0.7 \pm 0.1) \times 10^{-5}$ \\
$f_{4}^{Z}=0.2$ & $(1.5 \pm 0.2) \times 10^{-5}$ \\
$f_{5}^{Z}=0.025$ & $(1.9 \pm 0.3) \times 10^{-5}$ \\
$f_{5}^{Z}=0.05$ & $(2.0 \pm 0.3) \times 10^{-5}$ \\
$f_{5}^{Z}=0.1$ & \\
$f_{5}^{Z}=0.2$ &
\end{tabular}

procedure according to the $e^{+} e^{-} \rightarrow(Z / \gamma)^{*}(Z / \gamma)^{*} \rightarrow f \bar{f} f^{\prime} \bar{f}^{\prime}$ anomalous matrix element, either under off-shell [Eqs. (11)(18)] or on-shell [14] assumptions. Again, the relative differences in cross section between the two approaches are extremely small (Table II). Similarly to the $h_{j}^{Z}$ case, the mean values of the optimal observables give access to the values of the $f_{j}^{Z}$ couplings. The differences between off-shell and onshell treatments due to discrepancies in the shape of the phase space distributions are denoted by $\Delta f$. Figure 3 shows that the differences never exceed $|\Delta f|=0.015$, and are negligible when compared to the present experimental uncertainties [13].

In order to investigate the implications for the next generation of linear colliders, all previous exercises are repeated for a center-of-mass energy of $\sqrt{s}=500 \mathrm{GeV}$. Since the sensitivity at these energies is expected to be at least one order of magnitude larger than at $\sqrt{s}=200 \mathrm{GeV}$ [14], the values $h_{j}^{Z}, f_{j}^{Z}=0.025,0.05,0.1,0.2$ are considered. Cross section differences are shown in Table III, and the shifts due to shape distribution discrepancies are presented in Figs. 4 and 5. It is evident that the differences between off-shell and on-shell treatments are extremely small in all cases.

Finally, we should investigate the effect of changing the mass window cut around the $Z$ mass. This concerns the hypothetical case of a LEP analysis with relaxed constraints and also the $h_{j}^{V}$ limits obtained at Tevatron [3,4]. At $p \bar{p}$ colliders the requirements of consistency with the $Z$ mass are either loose (CDF) or somehow indirect (D0 and $Z \rightarrow \nu \bar{\nu}$ ). We have estimated the $h_{j}^{Z}$ and $f_{j}^{Z}$ differences between onshell and off-shell approaches for an invariant mass cut of 

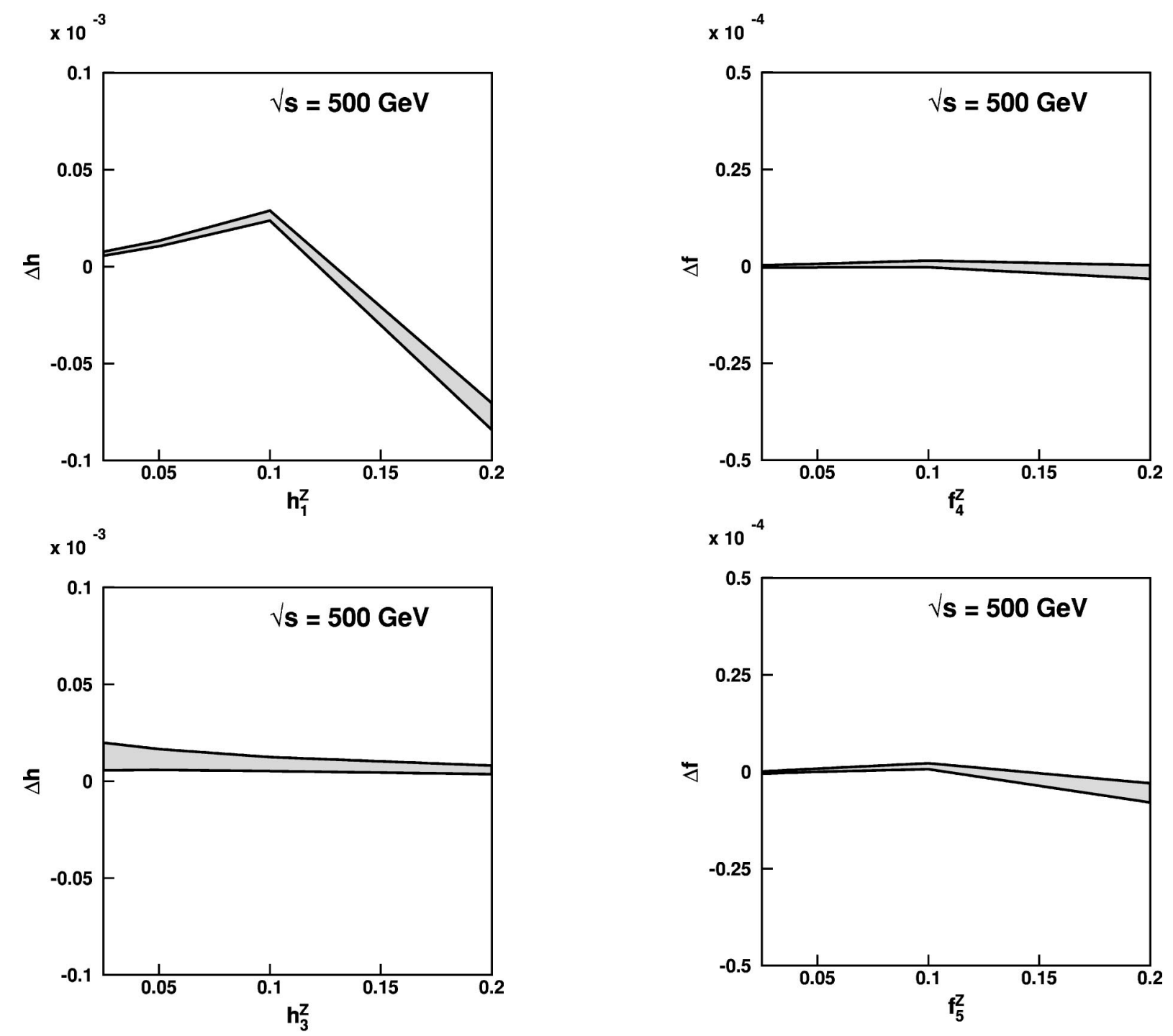

FIG. 4. Differences, $\Delta h$, between off-shell and on-shell measurements of the anomalous gauge couplings $h_{1}^{Z}$ (left) and $h_{3}^{Z}$ (right). Measurements are derived from the mean values of the $\mathrm{O}_{2}$ distribution (for $h_{1}^{Z}$ ) and of the $O_{1}$ distribution (for $h_{3}^{Z}$ ). The analyzed process is $e^{+} e^{-} \rightarrow(Z / \gamma)^{*} \gamma \rightarrow f \bar{f} \gamma$ at $\sqrt{s}=500 \mathrm{GeV}$.

$\left|m_{f \bar{f}}-m_{\mathrm{Z}}\right|<50 \mathrm{GeV}$. The results do not differ significantly from those obtained for $\left|m_{f \bar{f}}-m_{\mathrm{Z}}\right|<10 \mathrm{GeV}$. Table IV and Fig. 6 show them for the coupling where the largest effect is found $\left(f_{5}^{Z}\right)$. We conclude that the inclusion of final fermion pairs away from the $Z$ resonance region leads to marginal biases in the analysis.

So far, we have only considered the case in which $h_{j}^{V}$ and $f_{j}^{V}$ couplings are studied separately in $e^{+} e^{-} \rightarrow(Z / \gamma)^{*} \gamma$ and $e^{+} e^{-} \rightarrow(Z / \gamma)^{*}(Z / \gamma)^{*}$ events. However, once off-shell effects are included, combined searches may become a complicated issue. An example is the search for $f_{j}^{V}$ couplings in the $e^{+} e^{-} \rightarrow(Z / \gamma)^{*}(Z / \gamma)^{*}$ sample. In this case deviations due to the simultaneous presence of $h_{j}^{V}$ couplings (affecting the non-resonant $e^{+} e^{-} \rightarrow Z^{*} \gamma^{*}$ component) may arise.

Although tiny effects are expected in regions in which the
FIG. 5. Differences, $\Delta f$, between off-shell and on-shell measurements of the anomalous gauge couplings $f_{4}^{Z}$ (left) and $f_{5}^{Z}$ (right). Measurements are derived from the mean values of the $\mathrm{O}_{2}$ distribution (for $f_{4}^{Z}$ ) and of the $O_{1}$ distribution (for $f_{5}^{Z}$ ). The analyzed process is $e^{+} e^{-} \rightarrow(Z / \gamma)^{*}(Z / \gamma)^{*} \rightarrow f \bar{f} f^{\prime} \bar{f}^{\prime}$ at $\sqrt{s}=500 \mathrm{GeV}$.

$\left|M\left(e^{+} e^{-} \rightarrow Z^{*} \gamma^{*}\right) / M\left(e^{+} e^{-} \rightarrow Z^{*} Z^{*}\right)\right|$ matrix element ratio is small, a full off-shell treatment is advisable in general.

\section{AN ALTERNATIVE VIEW: REDEFINITION OF THE $\boldsymbol{h}_{2}^{V}$ AND $h_{4}^{V}$ CONVENTION}

Actually, the problem with the convention in Eqs. (1) and (2) can be solved at the "construction" level, just by imposing Bose-Einstein symmetry and electromagnetic gauge invariance as constraints.

Let us first consider the $f_{5}^{Z}$ case. What is relevant in the definition is the basic $P$-violating structure $i \epsilon^{\alpha \beta \mu \rho} q_{1 \rho}$. On it we have to impose Bose-Einstein symmetry for the three $Z$ bosons. It can be seen that a symmetrization of $i \epsilon^{\alpha \beta \mu \rho}\left(q_{1 \rho}\right.$ $-q_{2 \rho}$ ) leads to a trivial vanishing result. Therefore, one has to multiply it by a momentum-dependent scalar factor (cor- 
responding to a higher dimension term):

$$
\begin{aligned}
& \text { ie } f_{5}^{Z} \epsilon^{\alpha \beta \mu \rho}\left(q_{1 \rho}-q_{2 \rho}\right) \\
& \quad \rightarrow \text { ie } f_{5}^{Z} \frac{q_{3}^{2}}{m_{\mathrm{Z}}^{2}} \epsilon^{\alpha \beta \mu \rho}\left(q_{1 \rho}-q_{2 \rho}\right) .
\end{aligned}
$$

It is the symmetrization of this last expression ${ }^{4}$ which leads to the off-shell equation (11). A second example concerns the $h_{1}^{\gamma}$ coupling. In this case, we have to impose not only Bose-Einstein symmetry, but electromagnetic gauge invariance on the $P$-conserving term $i\left(q_{2}^{\mu} g^{\alpha \beta}-q_{2}^{\alpha} g^{\beta \mu}\right)$. This last requirement reads $q_{3 \mu} \Gamma_{\mathrm{Z} \gamma \gamma}^{\alpha \beta \mu}=q_{2 \beta} \Gamma_{\mathrm{Z} \gamma \gamma}^{\alpha \beta \mu}=0$, but, since terms proportional to $q_{2 \beta}, q_{3 \mu}$ are neglected, the right expressions to use are $q_{2 \beta} \Gamma_{\mathrm{Z} \gamma \gamma}^{\alpha \beta \mu} \propto q_{2}^{2}, q_{3 \mu} \Gamma_{\mathrm{Z} \gamma \gamma}^{\alpha \beta \mu} \propto q_{3}^{2}$. The two constraints are satisfied by the following modification:

$$
\begin{aligned}
& i e h_{1}^{\gamma}\left(q_{2}^{\mu} g^{\alpha \beta}-q_{2}^{\alpha} g^{\beta \mu}\right) \\
& \rightarrow i e h_{1}^{\gamma} \frac{q_{3}^{2}}{m_{\mathrm{Z}}^{2}}\left(q_{2}^{\mu} g^{\alpha \beta}-q_{2}^{\alpha} g^{\beta \mu}\right) .
\end{aligned}
$$

Again it is the symmetrization of this last expression which leads to the off-shell equation (18).

Let us now discuss the issue of anomalous couplings proceeding via higher dimension Lagrangians. Even if more offshell structures, not covered by $h_{2}^{V}$ and $h_{4}^{V}$ on-shell studies, are possible in this case [9], the experimental sensitivity to those new terms is extremely low. Besides the fact that they correspond to effects from terms of higher dimension, they vanish exactly for $Z, \gamma$ on-shell production, whereas a reasonable rate of off-shell boson production is required in order to perform a sensible measurement. Let us also recall that the most general parametrization used in the search for $W W V$ anomalous couplings [11] neglects terms vanishing in the on-shell limit.

Therefore, only terms associated in the on-shell limit to $h_{2}^{V}$ and $h_{4}^{V}$ structures will be considered. Imposing BoseEinstein symmetry and electromagnetic gauge invariance the following expressions are obtained:

$$
\begin{aligned}
& \Gamma_{\mathrm{Z} \gamma \mathrm{Z}}^{\alpha \beta \mu} \rightarrow i e \frac{h_{2}^{\mathrm{Z}}}{m_{\mathrm{Z}}^{4}}\left[q_{3}^{2} q_{3}^{\alpha}\left(q_{2} q_{3} g^{\beta \mu}-q_{2}^{\mu} q_{3}^{\beta}\right)\right. \\
&\left.+q_{1}^{2} q_{1}^{\mu}\left(q_{2} q_{1} g^{\alpha \beta}-q_{2}^{\alpha} q_{1}^{\beta}\right)\right]+i e \frac{h_{2}^{Z}}{2 m_{\mathrm{Z}}^{2}} \\
& \times\left[\left(q_{3}^{2}-q_{1}^{2}\right)\left(q_{2}^{\mu} g^{\alpha \beta}-q_{2}^{\alpha} g^{\beta \mu}\right)\right] \\
& \Gamma_{\mathrm{Z} \gamma \gamma}^{\alpha \beta \mu} \rightarrow i e \frac{h_{2}^{\gamma}}{m_{\mathrm{Z}}^{4}}\left[\left(q_{3}^{\alpha} q_{3}^{2}+q_{2}^{\alpha} q_{2}^{2}\right)\left(q_{2} q_{3} g^{\beta \mu}-q_{2}^{\mu} q_{3}^{\beta}\right)\right]
\end{aligned}
$$

\footnotetext{
${ }^{4}$ Scalar factors like $\left(q_{1}^{2}+q_{2}^{2}\right)$ and $\left(q_{1} q_{2}\right)$ lead to an equivalent
} result.

$$
\begin{gathered}
\Gamma_{\mathrm{Z} \gamma \mathrm{Z}}^{\alpha \beta \mu} \rightarrow i e \frac{h_{4}^{\mathrm{Z}}}{m_{\mathrm{Z}}^{4}}\left[q_{3}^{2} q_{3}^{\alpha} \epsilon^{\mu \beta \rho \sigma} q_{3 \rho} q_{2 \sigma}+q_{1}^{2} q_{1}^{\mu} \epsilon^{\alpha \beta \rho \sigma} q_{1 \rho} q_{2 \sigma}\right] \\
+i e \frac{h_{4}^{Z}}{2 m_{\mathrm{Z}}^{2}}\left[\left(q_{3}^{2}-q_{1}^{2}\right) \epsilon^{\alpha \beta \mu \rho} q_{2 \rho}\right]
\end{gathered}
$$

$\Gamma_{\mathrm{Z} \gamma \gamma}^{\alpha \beta \mu} \rightarrow i e \frac{h_{4}^{\gamma}}{m_{\mathrm{Z}}^{4}}\left[\left(q_{3}^{\alpha} q_{3}^{2}-q_{2}^{\alpha} q_{2}^{2}\right) \epsilon^{\mu \beta \rho \sigma} q_{3 \rho} q_{2 \sigma}\right]$

In the on-shell limit the corresponding structures in Eq. (1) are obtained. Let us comment at this point that imposing Bose-Einstein symmetry on the original proposal for $h_{2}^{Z}$ and $h_{4}^{Z}$ couplings forces the inclusion of redundant structures of the $h_{1}^{Z}$ and $h_{3}^{Z}$ type, as it can be easily confirmed by visual inspection of Eqs. (30) and (32).

\section{$S U(2)_{L} \times U(1)_{Y}$ SYMMETRY}

Given the good agreement between present data and SM predictions, any signal of new physics from a large scale $\Lambda$ will most probably manifest at the electroweak scale as deviations respecting the gauge symmetry of the SM. Concerning NTGCs, we must consider all terms containing neutral gauge bosons and Higgs fields in the linear realization of the $S U(2)_{L} \times U(1)_{Y}$ gauge symmetry. Eight operators manifest at the lowest dimension (eight):

$$
\begin{aligned}
& \mathcal{O}_{8}^{A}=i \widetilde{B}_{\mu \nu}\left(\partial_{\sigma} B^{\sigma \mu}\right)\left(\Phi^{+} D^{\nu} \Phi\right)+\text { H.c. } \\
& \mathcal{O}_{8}^{B}=i \widetilde{B}_{\mu \nu}\left(\partial_{\sigma} W_{I}^{\sigma \mu}\right)\left(\Phi^{+} \tau_{I} D^{\nu} \Phi\right)+\text { H.c. } \\
& \mathcal{O}_{8}^{C}=i \widetilde{W}_{I \mu \nu}\left(\partial_{\sigma} B^{\sigma \mu}\right)\left(\Phi^{+} \tau_{I} D^{\nu} \Phi\right)+\text { H.c. } \\
& \mathcal{O}_{8}^{D}=i \widetilde{W}_{I \mu \nu}\left(\partial_{\sigma} W_{I}^{\sigma \mu}\right)\left(\Phi^{+} D^{\nu} \Phi\right)+\text { H.c. } \\
& \widetilde{\mathcal{O}}_{8}^{A}=i B_{\mu \nu}\left(\partial_{\sigma} B^{\sigma \mu}\right)\left(\Phi^{+} D^{\nu} \Phi\right)+\text { H.c. } \\
& \widetilde{\mathcal{O}}_{8}^{B}=i B_{\mu \nu}\left(\partial_{\sigma} W_{I}^{\sigma \mu}\right)\left(\Phi^{+} \tau_{I} D^{\nu} \Phi\right)+\text { H.c. } \\
& \widetilde{\mathcal{O}}_{8}^{C}=i W_{I \mu \nu}\left(\partial_{\sigma} B^{\sigma \mu}\right)\left(\Phi^{+} \tau_{I} D^{\nu} \Phi\right)+\text { H.c. } \\
& \widetilde{\mathcal{O}}_{8}^{D}=i W_{I \mu \nu}\left(\partial_{\sigma} W_{I}^{\sigma \mu}\right)\left(\Phi^{+} D^{\nu} \Phi\right)+\text { H.c. }
\end{aligned}
$$

where $B^{\mu \nu}$ and $W_{I}^{\mu \nu}(I=1,3)$ are the tensor field associated to the $U(1)_{Y}$ and $S U(2)_{L}$ groups, respectively, $\tau_{I}$ are the Pauli matrices, $\Phi$ is the Higgs field and $D$ denotes the covariant derivative. The first four operators conserve $C P$ and the last four operators are $C P$-violating.

These eight operators give independent contributions to the $h_{1}^{V}, h_{3}^{V}, f_{4}^{V}, f_{5}^{V}$ couplings discussed in previous sections. Therefore, no $S U(2)_{L} \times U(1)_{Y}$ constraints among NTGC couplings can be imposed. Even under the extreme assumption of fully vanishing $C$-violating $W W V$ couplings, $g_{4}^{Z}, g_{5}^{Z}, g_{4}^{\gamma}, g_{5}^{\gamma}[11]$, constraints among NTGCs are weak, since these four charged couplings compete with eight different neutral effects. It can be shown that operators $\mathcal{O}_{8}^{A}$ and 
TABLE IV. Relative difference in the number of expected events, $\Delta N / N$, between off-shell and on-shell analyses at $\sqrt{s}=200$, $500 \mathrm{GeV}$. Different values of the $f_{5}^{Z}$ anomalous coupling are considered. The cut on the fermion-pair invariant masses is $\mid m_{f \bar{f}}$ $-m_{\mathrm{Z}} \mid<50 \mathrm{GeV}$.

\begin{tabular}{lcc}
\hline \hline Coupling value & $\sqrt{s}(\mathrm{GeV})$ & $\frac{\Delta N}{N}$ \\
\hline$f_{5}^{Z}=0.25$ & & $(7.1 \pm 0.9) \times 10^{-5}$ \\
$f_{5}^{Z}=0.5$ & 200 & $(2.0 \pm 0.2) \times 10^{-4}$ \\
$f_{5}^{Z}=1.0$ & 200 & $(6.0 \pm 0.4) \times 10^{-4}$ \\
$f_{5}^{Z}=2.0$ & 200 & $(1.6 \pm 0.1) \times 10^{-5}$ \\
$f_{5}^{Z}=0.025$ & 200 & $(0.8 \pm 0.1) \times 10^{-5}$ \\
$f_{5}^{Z}=0.05$ & 500 & $(1.6 \pm 0.2) \times 10^{-5}$ \\
$f_{5}^{Z}=0.1$ & 500 & $(2.0 \pm 0.3) \times 10^{-5}$ \\
$f_{5}^{Z}=0.2$ & 500 & $(2.1 \pm 0.3) \times 10^{-5}$ \\
\hline \hline
\end{tabular}
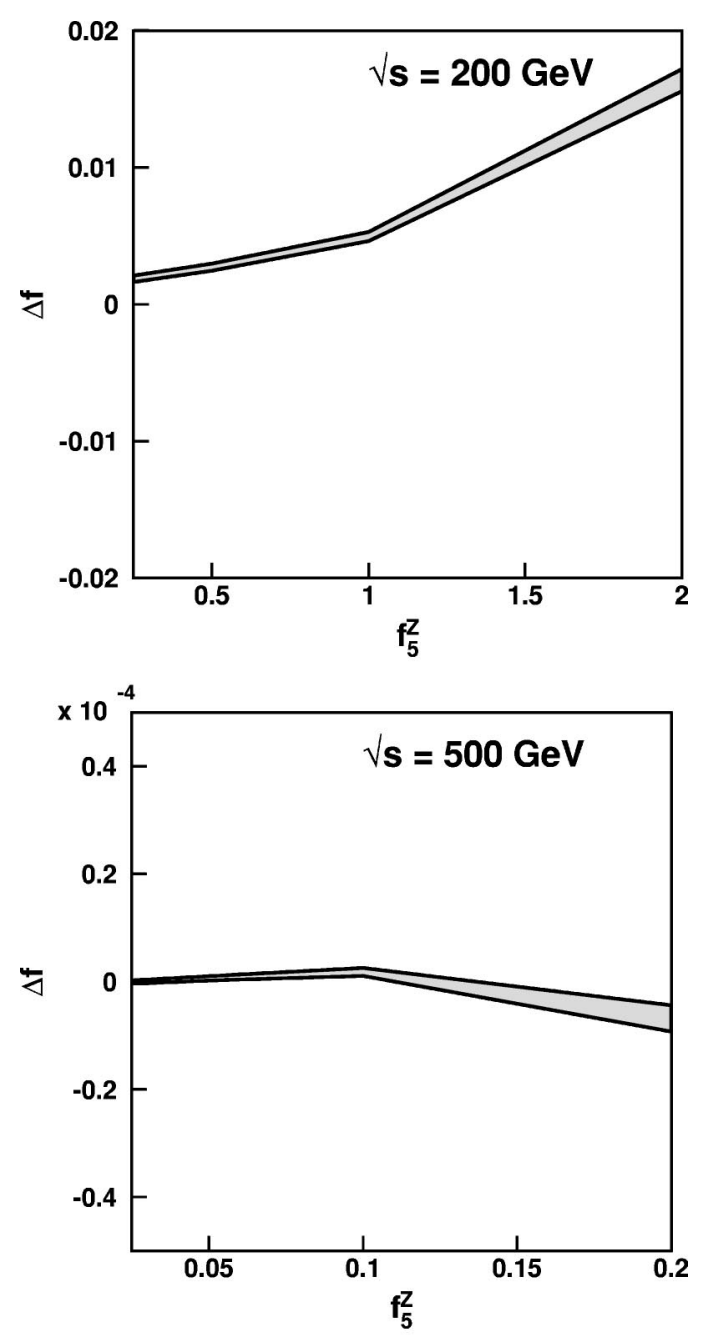

FIG. 6. Differences, $\Delta f$, between off-shell and on-shell measurements of the anomalous gauge coupling $f_{5}^{Z}$ at $\sqrt{s}=200 \mathrm{GeV}$ and $\sqrt{s}=500 \mathrm{GeV}$. Measurements are derived from the mean value of the $O_{1}$ distribution. The analyzed process is $e^{+} e^{-}$ $\rightarrow(Z / \gamma)^{*}(Z / \gamma)^{*} \rightarrow f \bar{f} f^{\prime} \bar{f}^{\prime}$. A loose invariant mass cut, $\left|m_{f \bar{f}}-m_{\mathrm{Z}}\right|$ $<50 \mathrm{GeV}$, is used.
$\widetilde{\mathcal{O}}_{8}^{A}$ do not contain $W W V$ couplings, whereas the operators $\mathcal{O}_{8}^{B}$ and $\widetilde{\mathcal{O}}_{8}^{B}$ have no effect on $W W V$ couplings for on-shell $W$ bosons. This last feature follows trivially from the on-shell relation: $\partial_{\sigma} W^{\sigma \mu}=-m_{\mathrm{W}}^{2} W^{\mu}$. If only $\mathcal{O}_{8}^{A}, \mathcal{O}_{8}^{B}, \widetilde{\mathcal{O}}_{8}^{A}$ and $\widetilde{\mathcal{O}}_{8}^{B}$ are allowed, then the following constraints among NTGCs are found:

$$
\begin{aligned}
& f_{5}^{Z}=h_{3}^{Z} \tan \theta_{w} \\
& f_{5}^{\gamma}=h_{3}^{\gamma} \tan \theta_{w} \\
& f_{4}^{Z}=h_{1}^{Z} \tan \theta_{w} \\
& f_{4}^{\gamma}=h_{1}^{\gamma} \tan \theta_{w}
\end{aligned}
$$

where $\theta_{w}$ is the Weinberg angle. Our conclusions are different from those of Ref. [9], where only operators containing exclusively neutral gauge bosons and Higgs fields are considered as relevant and strong constraints among NTGCs are presented.

\section{CONCLUSIONS}

We have analyzed the experimental consequences of including a proper off-shell treatment in the searches for anomalous NTGCs. We find that the quantitative differences between on-shell and off-shell treatments are negligible, provided that the $e^{+} e^{-} \rightarrow Z \gamma$ and $e^{+} e^{-} \rightarrow Z Z$ analyses are performed in regions where $Z$ resonant production is dominant. This conclusion is also valid for future $e^{+} e^{-}$studies at higher energies. Present on-shell studies guarantee a coverage of all physics deviations for which a reasonable experimental sensitivity is expected. Just for theoretical consistency, and in order to avoid misleading results in offresonance studies, we advocate the use of the new vertex functions presented in Eqs. (11)-(18) and (30)-(33). Contrary to what has been recently suggested in the literature [9], we find that only the additional assumption of vanishing $C$-violating charged gauge couplings in the $e^{+} e^{-} \rightarrow W^{+} W^{-}$ process may lead to some $S U(2)_{L} \times U(1)_{Y}$ constraints among NTGCs.

\section{ACKNOWLEDGMENTS}

We would like to thank Helge Voss and Robert Sekulin for interesting discussions and suggestions concerning the paper and the application of $S U(2) \times U(1)$ constraints at LEP. J.A. was partially supported by CICYT Grant No. AEN99-0305. 
[1] T. Barklow et al., Anomalous Gauge Boson Couplings, Proceedings of the 1996 DPF/DPB Summer Study on New Directions in High-Energy Physics, Snowmass, Colorado, hep-ph/9611454.

[2] G.J. Gounaris, J. Layssac, and F.M. Renard, Phys. Rev. D 62, 073013 (2000).

[3] CDF Collaboration, F. Abe et al., Phys. Rev. Lett. 74, 1941 (1995).

[4] D0 Collaboration, S. Abachi et al., Phys. Rev. Lett. 78, 3640 (1997); D0 Collaboration, B. Abbott et al., Phys. Rev. D 57, 3817 (1998).

[5] ALEPH Collaboration, ALEPH 2001-061 CONF 2001-041, 2001.

[6] DELPHI Collaboration, DELPHI 2001-097 CONF 525, 2001.

[7] L3 Collaboration, M. Acciarri et al., Phys. Lett. B 436, 187 (1999); L3 Collaboration, M. Acciarri et al., ibid. 489, 55 (2000); L3 Collaboration, L3 Note 2672, 2001; L3 Collaboration, M. Acciarri et al., Phys. Lett. B 450, 281 (1999); L3 Collaboration, M. Acciarri et al., ibid. 465, 363 (1999); L3
Collaboration, L3 Note 2696, 2001.

[8] OPAL Collaboration, G. Abbiendi et al., Eur. Phys. J. C 17, 13 (2000); OPAL Collaboration, G. Abbiendi et al., Phys. Lett. B 476, 256 (2000); OPAL Collaboration, OPAL PN482, 2001.

[9] G.J. Gounaris, J. Layssac, and F.M. Renard, Phys. Rev. D 62, 073012 (2000).

[10] D. Choudhury, S. Dutta, S. Rakshit, and S. Rindani, Int. J. Mod. Phys. A 16, 4891 (2001).

[11] K. Hagiwara, K. Hikasa, R.D. Peccei, and D. Zeppenfeld, Nucl. Phys. B282, 253 (1987).

[12] G.J. Gounaris, J. Layssac, and F.M. Renard, Phys. Rev. D 61, 073013 (2000).

[13] The LEP Collaborations and the LEP GC Working Group, LEPEWWG/TGC/2001-03, ALEPH 2001-072 PHYSIC 2001022, DELPHI 2001-127 PHYS 903, L3 Note 2710, OPAL TN703, 2001.

[14] J. Alcaraz, M.A. Falagán, and E. Sánchez, Phys. Rev. D 61, 075006 (2000). 